Vol. 6 (1997): 247-258.

\title{
Classification of acid sulphate soils of Finland according to Soil Taxonomy and the FAO/Unesco legend
}

\author{
Markku Yli-Halla \\ Agricultural Research Centre of Finland, Institute of Resource Management, FIN-31600 Jokioinen, Finland, \\ e-mail: markku.yli-halla@mtt.fi
}

\begin{abstract}
In order to place acid sulphate soils (a.s. soils) of Finland in an international context, five pedons from cultivated a.s. soils from Liminka, Ylistaro and Laitila on the western coast of Finland were analyzed and classified according to Soil Taxonomy and the revised legend of the FAO/Unesco Soil Map of the World. Three of the pedons (Liminka 1 and 2, Laitila 1) had sulfuric horizons within 50 $\mathrm{cm}$ of soil surface and qualify as Typic Sulfaquepts. One pedon (Ylistaro) had a sulfuric horizon at the depth of 100-150 cm and was classified as a Sulfic Cryaquept. The fifth pedon (Laitila 2) did not have either a sulfuric horizon or sulfidic materials, but it had a $\mathrm{pH}<4.0$ and enough $\mathrm{SO}_{4}-\mathrm{S}$ to be classified as a Sulfic Cryaquept. According to the FAO/Unesco legend, all pedons were classified as Thionic Gleysols. Thus Typic Sulfaquepts and Sulfic Cryaquepts (Soil Taxonomy) and Thionic Gleysols (FAO/Unesco classification) occur commonly on the western coast of Finland. As a result of artificial drainage and leaching, they gradually fail to meet the requirements of a.s. soil classes and will be classified as Typic Cryaquepts. In terms of the FAO/Unesco nomenclature, Thionic Gleysols become Dystric Gleysols or Gleyic Cambisols over time.
\end{abstract}

Key words: FAO/Unesco Soil Map of the World, pedogenesis, soil morphology, soil pH, soil survey, Soil Taxonomy, sulphur content

\section{Introduction}

Acid sulphate soils (a.s. soils) are soils in which, "as a result of processes of soil formation, sulphuric acid either will be produced, is being produced or has been produced in amounts that have a lasting effect on main soil characteristics" (Pons 1973). A more quantitative criterion, needed for soil classification, has been presented, e.g. by van Mensvoort and Dent (1997): In a.s. soils, enough sulphuric acid is produced to bring soil $\mathrm{pH}$ below 4 . In the revised legend to the FAO/ Unesco Soil Map of the World (FAO 1988), these soils are called Thionic Gleysols, Thionic Fluvisols or Thionic Histosols. In Soil Taxonomy (Soil Survey Staff 1996), a.s. soils are dispersed through several taxa. The most coherent and detailed classification of a.s. soils by Dent (1986) makes the important distinction between 'sulphidic soils' (or potential a.s. soils), 'raw a.s. soils' that are actively generating sulphuric acid 


\section{Yli-Halla, M. Acid sulphate soils of Finland}

and which, therefore, pose an environmental hazard, and 'ripe acid sulphate soils' that are still severely acid ( $\mathrm{pH} 4$ or less) but are no longer exporting acidity. The 1994 revision of Soil Taxonomy (Fanning and Witty 1993) seeks to distinguish the 'raw acid sulphate soils' as Sulfaquepts using a severe $\mathrm{pH}$ criterion $(\mathrm{pH}<3.5)$ for the diagnostic sulfuric horizon.

The a.s. soils of Finland, mainly located on the coast of the Gulf of Bothnia, have developed in sulfidic sediment accumulated during the Litorina period of the Baltic sea $(7000-3000 \mathrm{yr}$ B.P.). These soils were earlier studied mainly from the agricultural point of view (Kivinen 1938, Purokoski 1958, 1959, Erviö 1975, Erviö and Palko 1984). Since the 1980's, the emphasis has been in environmental aspects, particularly acid loading from these soils to surface waters (Palko et al. 1985, 1987, Palko and Saari 1987, Hartikainen and Yli-Halla 1986, Palko 1988, 1994, Erviö 1991, Palko and Yli-Halla 1993). Acid sulphate soils of Finland have not been classified according to international systems, but local criteria have been applied to identify a.s. soils in national soil surveys. In Finland, Erviö (1975) and Erviö and Palko (1984) called a soil an a.s. soil if the subsoil $(40-60 \mathrm{~cm}$ or $50-70 \mathrm{~cm})$ had a $\mathrm{pH}\left(\mathrm{H}_{2} \mathrm{O}, 1: 2.5\right)<5.0$ and/or a $\mathrm{SO}_{4}-\mathrm{S}$ content $>100$ $\mathrm{mg} \mathrm{l}^{-1}$ of soil, determined in air-dried samples. More recently (Palko and Saari 1987, Palko et al. 1987), the use of $\mathrm{SO}_{4}-\mathrm{S}$ content as the criterion for classification was discontinued and the
$\mathrm{pH}$ was determined in the field in a moist soil. The minimum $\mathrm{pH}(<5.0)$ of the profile was the principal criterion in the classification, and redox potential was a secondary variable.

The purpose of this paper is to place the a.s. soils of Finland in an international context. Five representative pedons from three a.s. soil areas on the western coast of Finland are classified according to Soil Taxonomy and the revised legend to the FAO/Unesco Soil Map of the World.

\section{Acid sulphate soils in Soil Tax- onomy and $\mathrm{FAO} /$ Unesco legend}

A sulfuric horizon and sulfidic materials (Table 1) are essential in the classification of a.s. soils. In Soil Taxonomy (Soil Survey Staff 1996), the determination of sulfidic materials is based on soil $\mathrm{pH}$ before and after aerobic incubation. Sulfidic materials are stable in anaerobic environments. A sulfuric horizon develops when sulfidic materials are oxidized to sulfuric acid, resulting in a sufficient decrease of $\mathrm{pH}$. In Soil Taxonomy, evidence that the acidity is caused by sulfuric acid is required. Accepted evidence is at least one of the following: 1) presence of jarosite, 2) high content of water-soluble $\mathrm{SO}_{4}-\mathrm{S}$ in the horizon concerned and 3) sulfidic materi-

Table 1. Characteristics of sulfidic materials and the sulfuric horizon.

\begin{tabular}{lll}
\hline $\begin{array}{l}\text { Taxonomic } \\
\text { feature }\end{array}$ & Soil Taxonomy & $\begin{array}{l}\text { FAO/Unesco Soil } \\
\text { Map of the World }\end{array}$ \\
\hline Sulfidic materials & $\begin{array}{l}\mathrm{pH}>3.5 . \text { After incubation } \mathrm{pH} \text { decreases } \\
\text { by at least } 0.5 \text { units and reaches a } \mathrm{pH}<4 .\end{array}$ & $\begin{array}{l}\text { Total } \mathrm{S}>0.75 \% \text { mostly in the form of sulfide } \\
\text { and }>3.5 . \text { After drainage pH decreases } \\
\text { below } 3.5 .\end{array}$ \\
Sulfuric horizon & $\begin{array}{l}\mathrm{pH} \leq 3.5 \text { and thickness at least } 15 \mathrm{~cm} \\
\text { and one or more of the following: } \\
\text { - jarosite concentrations }\end{array}$ & $\begin{array}{l}\mathrm{pH}<3.5 \text { and thickness at least } 15 \mathrm{~cm} \text { and } \\
\text { generally jarosite mottles }\end{array}$ \\
& $\begin{array}{l}\text { - directly underlying sulfidic materials } \\
\text { - water-soluble } \mathrm{SO}_{4}-\mathrm{S}>0.05 \%\end{array}$ & \\
\hline
\end{tabular}


Vol. 6 (1997): 247-258.

Table 2. General requirements of some acid sulphate soil units according to Soil Taxonomy (Soil Survey Staff 1996) and the legend of the FAO/Unesco Soil Map of the World (FAO 1988).

\begin{tabular}{ll}
\hline Soil unit & Description \\
\hline $\begin{array}{l}\text { Soil Taxonomy: } \\
\text { Sulfaquents }\end{array}$ & $\begin{array}{l}\text { Entisols with aquic soil moisture regimes and sulfidic materials within } 50 \mathrm{~cm} \text { of } \\
\text { soil surface } \\
\text { Inceptisols with aquic soil moisture regimes and sulfuric horizons within } 50 \mathrm{~cm} \\
\text { of soil surface } \\
\text { Inceptisols with aquic soil moisture regimes and cryic temperature regimes and } \\
\text { with one or more of the following: } \\
\text { Sulfic Cryaquepts }\end{array}$ \\
& - a sulfuric horizon between 50 and $150 \mathrm{~cm}$ from soil surface \\
& - within $150 \mathrm{~cm}$ of soil surface a horizon which has all the characteristics of \\
& a sulfuric horizon except pH which is between 3.5 and 4. \\
& $\begin{array}{l}\text { Gleyic properties within } 0.5 \mathrm{~m} \text { of soil surface. } \\
\text { Thionic Gleysols }\end{array}$ \\
& $\begin{array}{l}\text { Sulfuric horizons or sulfidic materials within } 125 \mathrm{~cm} \\
\text { of soil surface }\end{array}$ \\
\hline
\end{tabular}

als below the acidic horizon. A sulfuric horizon must have a $\mathrm{pH} \leq 3.5$. This requirement attempts to include horizons where sulfide is presently oxidizing (raw acid sulphate soils in Dent's classification) and to exclude horizons where all sulfide has already disappeared (Fanning and Witty 1993). The legend of the FAO/Unesco Soil Map of the World (FAO 1988) uses merely the oxidized $\mathrm{pH}$ value which must be $<3.5$.

The essence of the taxonomic definitions of the a.s. soils is to include soils which have enough sulfuric acid to exhibit a $\mathrm{pH}<3.5$ (FAO 1988 ) or $<4.0$ (Soil Survey Staff 1996), or enough sulfide to produce, upon oxidation, a pH below these values. In Soil Taxonomy, a.s. soil units include great groups in Entisols, Inceptisols and Histosols, e.g. Sulfaquents, Sulfaquepts and Sulfohemists, respectively, and Sulfic subgroups in Entisols and Inceptisols, e.g. Sulfic Endoaquents and Sulfic Cryaquepts, respectively. In that system, soils in which sulfide is oxidizing and reduced soils which contain sulfidic materials but not an acidic horizon are classified into different units. In the revised legend of the Soil Map of the World (FAO 1988), thionic units are recognized in Histosols, Fluvisols, Gleysols and soils with a sulfuric horizon and those with only sulfidic materials are classified in the same taxa. The requirements of some relevant a.s. soil units are summarized in Table 2.

\section{Material and methods}

Five pedons representative of the range of a.s. soils in Finland were described from Liminka (two pedons), Ylistaro and Laitila (two pedons) in Northern and Southern Ostrobothnia and Southwestern Finland, respectively (Fig. 1).

In Liminka, the study area $\left(64^{\circ} 50\right.$ ' $\mathrm{N}$, $25^{\circ} 24^{\prime} \mathrm{E}$ ) is flat and about $1 \mathrm{~m}$ above the level of the Gulf of Bothnia. It rose above the sea level 100-150 years ago. The Liminka 1 pedon (Fig. 2) has been cultivated for grass and small grains for about 15 years, but was fallowed at the time of inspection. Initially, the field had open drains, but it was pipe-drained 8 years prior to inspection. The ground water was not encountered within $1 \mathrm{~m}$ of soil surface on August 13, 1992 (sampling). The Liminka 2 pedon comes from an experimental polder (described in detail by Palko 1988) which was constructed 8 years be- 
Yli-Halla, M. Acid sulphate soils of Finland

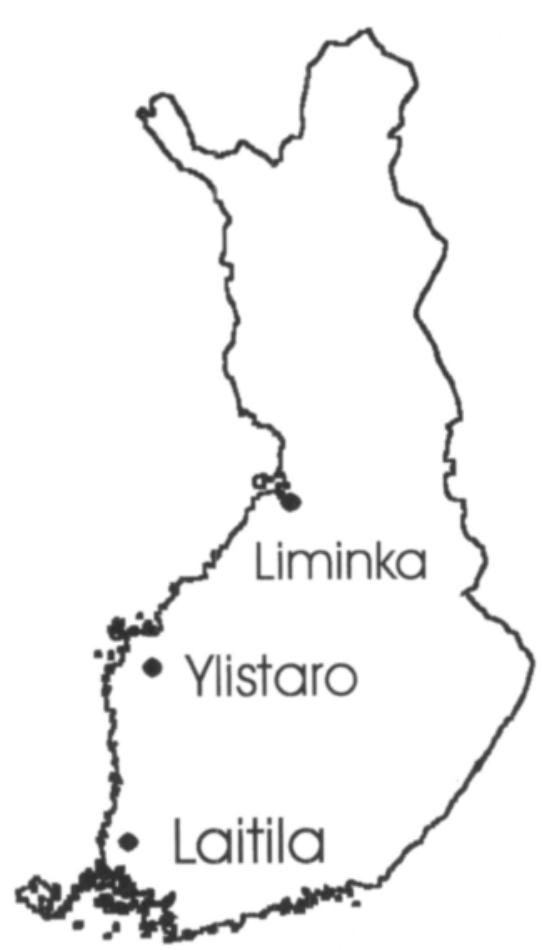

Fig. 1. Locations of the pedons.

fore sampling. At the same time, the sampled plot was pipe-drained to the depth of $113 \mathrm{~cm}$ and limed (15 tons dolomitic limestone ha $\left.{ }^{-1}\right)$. Oats had been grown in the field for three years, after which cropping was abandoned and the area was now covered with a grassy vegetation. Ground water was at $95 \mathrm{~cm}$ below the soil surface on August 14, 1992.

The Ylistaro pedon $\left(62^{\circ} 55^{\prime} \mathrm{N}, 2^{\circ} 29^{\prime} \mathrm{E}\right)$ represents the major acid sulphate soil area of Finland (Purokoski 1958, Erviö 1975). The pedon (Fig. 3) is located on a flat coastal plain with an elevation of $26 \mathrm{~m}$ asl. The area had been cultivated for about 100 years but has been fallow since 1991. Different grasses grow on the area now. Pipe drainage has been installed. No water table was encountered above $150 \mathrm{~cm}$ on October 3, 1996.

The Laitila pedons $\left(60^{\circ} 53\right.$ ' $\left.\mathrm{N}, 21^{\circ} 41^{\prime} \mathrm{E}\right)$ are located in a cultivated Valkojärvi polder (1000 ha) $10 \mathrm{~m}$ asl. The a.s. area, described by Palko et al. (1985), is drained to the River Sirppujoki by pumping. During the Litorina period the area was a gulf of the sea and later it became a lake. Lake Valkojärvi was drained for agricultural use piecemeal between the 1930's and 1966. Small grain and sugarbeet have been grown. The area of the Laitila 1 pedon was drained about 30 years ago. The site is the lowest-lying spot of the Valkojärvi polder. No groundwater was encountered on September 24, 1996. The Laitila 2 pedon is about $200 \mathrm{~m}$ away from the Laitila 1 pedon. Ground water ( $\mathrm{pH} 3.5$ ) was $95 \mathrm{~cm}$ below the soil surface on September 15, 1992.

In all pedons, moist Munsell colours were recorded. Where mollic/umbric epipedons were considered, also dry colours were determined. Each horizon was sampled, and samples air-dried and ground to pass a 2-mm sieve prior to analysis. In the Liminka 1 and 2 and Laitila 2 pedons, redox potential and soil $\mathrm{pH}$ were determined in the field in the soil pit at intervals of $10 \mathrm{~cm}$ by inserting electrodes directly into the soil. A small amount of deionized water was added to allow proper contact between the soil and the electrode (Puustinen et al. 1994). The samples from the Laitila 1 and Ylistaro pedons were transported to the laboratory in plastic bags, and the $\mathrm{pH}(1: 1)$ of the moist samples was determined the day after sampling. The $\mathrm{pH}$ of all soil samples was also measured after aerobic incubation and drying. Particle size distribution of mineral material was determined by a pipette method. The total contents of $\mathrm{C}, \mathrm{N}$ and $\mathrm{S}$ were determined using a Leco dry combustion apparatus (CNS1000). Because the samples were acid, all $C$ was assumed to be organic. After incubation for 2 months, $\mathrm{SO}_{4}$-S was extracted with $0.01 \mathrm{M} \mathrm{CaCl}_{2}$ and determined by Inductively Coupled Plasma Emission Spectrometry (ICP). Exchangeable cations $(\mathrm{Ca}, \mathrm{Mg}, \mathrm{K}, \mathrm{Na})$ were extracted with $1 \mathrm{M} \mathrm{NH}_{4}$-acetate at $\mathrm{pH} 7$. Acidity released from the soil was determined by titrating the soil extract back to the original $\mathrm{pH}$ with $0.02 \mathrm{M} \mathrm{NaOH}$. The sum of $\mathrm{K}, \mathrm{Na}, \mathrm{Ca}, \mathrm{Mg}$ and acidity was taken as the cation exchange capacity (CEC) and the base saturation was calculated as the percentage of $\mathrm{Ca}+\mathrm{Mg}+\mathrm{K}+\mathrm{Na}$ of CEC. Free acid, present 


\section{AGRICULTURAL AND FOOD SCIENCE IN FINLAND}

Vol. 6 (1997): 247-258.

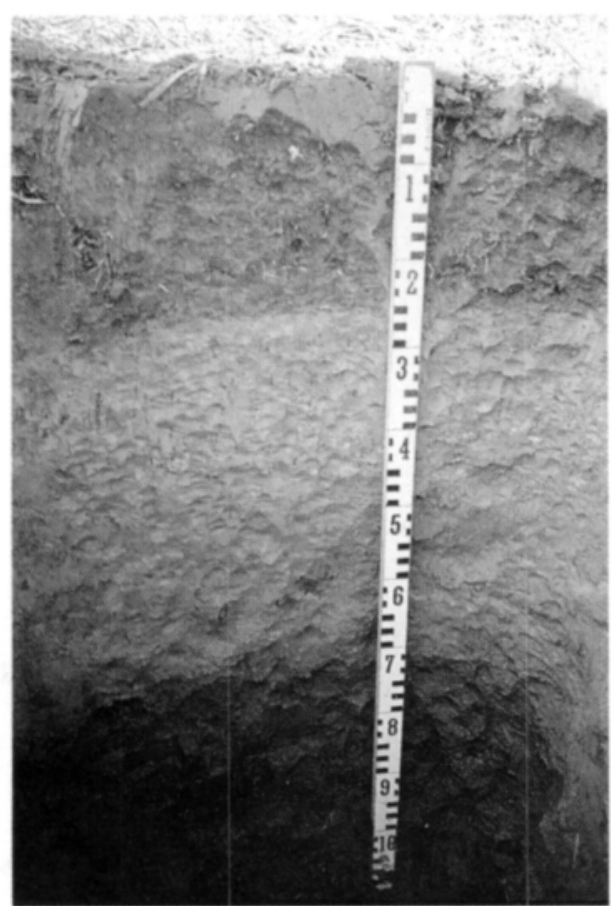

Fig. 2. The Liminka 1 pedon.

originally in the soil or formed upon drying and partial oxidation of sulfide, probably results in a slight overestimation of CEC and underestimation of base saturation in the most acid $(\mathrm{pH}<3.5)$ horizons and in the horizons containing sulfidic materials. Possible presence of free sulphate salts also adds to the overestimation of CEC. Poorly crystalline $\mathrm{Fe}$ and $\mathrm{Al}$ (hydr)oxides were extracted with $0.2 \mathrm{M} \mathrm{NH}_{4}$-oxalate ( $\mathrm{pH} 3.0$ ) in the dark (McKeague and Day 1966). Iron and $\mathrm{Al}$ were determined by ICP.

\section{Results}

All the soils were mineral soils. The organic C content was usually highest in the Ap horizons (Table 3), but the deeper layers were also richer in organic $\mathrm{C}$ than mineral soils in general, par-

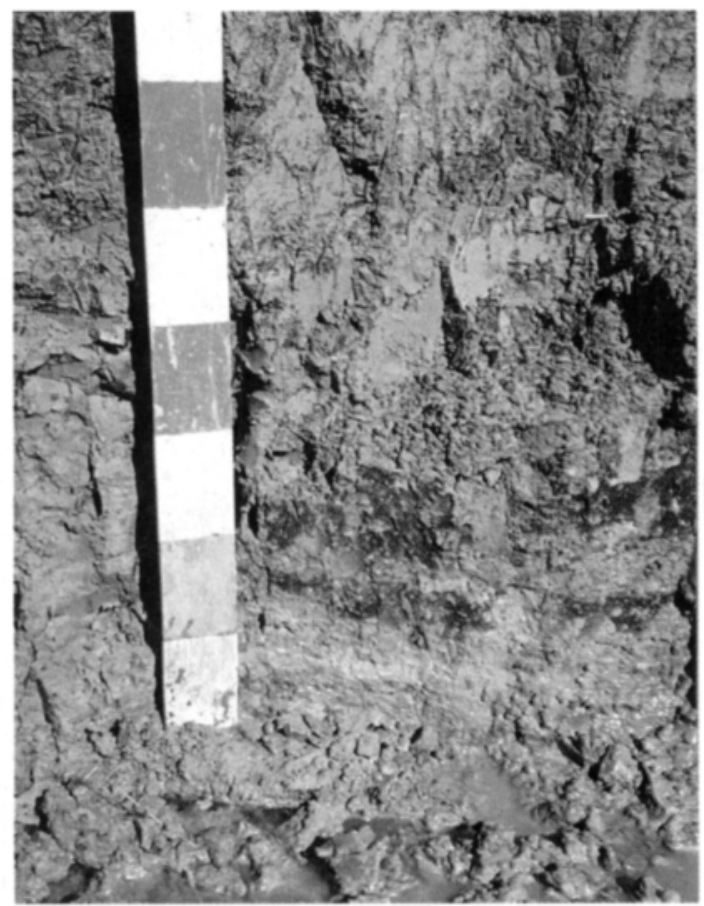

Fig. 3. The Ylistaro pedon from $0.80 \mathrm{~m}$ below.

ticularly in the Laitila pedons. The Laitila 1 pedon had the highest $\mathrm{C}$ content at the depth of $35-50 \mathrm{~cm}$, presumably inherited from the initial sediment. The pedons represent the following particle size classes:

$\begin{array}{lll}\text { Pedon } & \begin{array}{l}\text { Soil } \\ \text { Taxonomy }\end{array} & \begin{array}{l}\text { FAO/ } \\ \text { Unesco legend }\end{array} \\ \begin{array}{l}\text { Liminka 1 } \\ \text { and 2 }\end{array} & \begin{array}{l}\text { coarse silty } \\ \text { fine silty }\end{array} & \begin{array}{l}\text { medium textured } \\ \text { medium textured }\end{array} \\ \begin{array}{l}\text { Laitila 1 } \\ \text { and 2 }\end{array} & \text { fine } & \text { fine textured }\end{array}$

Based on the morphological descriptions (Table 4), it can be concluded that all pedons had ochric epipedons. Relatively high $\mathrm{pH}$ values in the Ap horizons of all pedons except Ylistaro (Table 5) are attributable to liming. All pedons had cambic B horizons (as indicated by the development of structure) and aquic moisture re- 


\section{AGRICULTURAL AND FOOD SCIENCE IN FINLAND}

Yli-Halla, M. Acid sulphate soils of Finland

Table 3. Selected physical and chemical properties of the pedons. ${ }^{1)}$

\begin{tabular}{|c|c|c|c|c|c|c|c|c|c|}
\hline $\begin{array}{l}\text { Depth } \\
\mathrm{cm}\end{array}$ & $\begin{array}{l}\text { Clay } \\
\%\end{array}$ & $\begin{array}{l}\text { Silt } \\
\%\end{array}$ & $\begin{array}{l}\mathrm{C} \\
\%\end{array}$ & $\begin{array}{l}S_{\text {tot }} \\
\%\end{array}$ & $\begin{array}{l}\mathrm{N}_{\text {tot }} \\
\%\end{array}$ & $\begin{array}{l}\text { CEC } \\
\mathrm{cmol}(+) / \mathrm{kg}\end{array}$ & $\begin{array}{l}\text { Base satu- } \\
\text { ration, \% }\end{array}$ & $\begin{array}{l}\mathrm{Fe}^{2)} \\
\mathrm{g} / \mathrm{kg}\end{array}$ & $\begin{array}{l}\mathrm{Al}^{2)} \\
\mathrm{g} / \mathrm{kg}\end{array}$ \\
\hline \multicolumn{10}{|c|}{ Liminka 1} \\
\hline $0-25$ & 10 & 82 & $2.9^{b}$ & $0.19^{\mathrm{a}}$ & $0.19^{b}$ & 17.5 & 89 & $10.1^{\mathrm{d}}$ & $1.7^{\mathrm{d}}$ \\
\hline $25-35$ & 8 & 86 & $0.4^{a}$ & $0.17^{\mathrm{a}}$ & $0.04^{\mathrm{a}}$ & 7.1 & 53 & $4.8^{\mathrm{ab}}$ & $0.5^{\mathrm{a}}$ \\
\hline $35-45$ & 10 & 86 & $0.4^{\mathrm{a}}$ & $0.27^{\mathrm{b}}$ & $0.04^{\mathrm{a}}$ & 8.8 & 46 & $3.8^{\mathrm{a}}$ & $0.5^{\mathrm{a}}$ \\
\hline $45-65$ & 10 & 85 & $0.6^{a}$ & $0.26^{\mathrm{b}}$ & $0.06^{\mathrm{a}}$ & 13.1 & 44 & $6.0^{\mathrm{b}}$ & $0.5^{\mathrm{a}}$ \\
\hline $65-85$ & 12 & 86 & $0.7^{\mathrm{a}}$ & $0.45^{d}$ & $0.06^{\mathrm{a}}$ & 15.5 & 48 & $6.0^{b}$ & $1.1^{\mathrm{c}}$ \\
\hline $85-100$ & 13 & 86 & $0.7^{\mathrm{a}}$ & $0.36^{c}$ & $0.06^{\mathrm{a}}$ & 13.7 & 80 & $8.0^{c}$ & $0.6^{\mathrm{b}}$ \\
\hline \multicolumn{10}{|c|}{ Liminka 2} \\
\hline $0-25$ & 11 & 83 & $6.0^{b}$ & $0.29^{\mathrm{a}}$ & $0.28^{\mathrm{b}}$ & 29.6 & 81 & $8.5^{b}$ & $1.6^{\mathrm{c}}$ \\
\hline $25-65$ & 9 & 85 & $0.5^{\mathrm{a}}$ & $0.24^{\mathrm{a}}$ & $0.05^{\mathrm{a}}$ & 11.6 & 41 & $5.7^{\mathrm{a}}$ & $0.6^{\mathrm{a}}$ \\
\hline $65-72$ & 10 & 80 & $0.6^{\mathrm{a}}$ & $0.66^{b}$ & $0.06^{\mathrm{a}}$ & 18.2 & 32 & $6.0^{\mathrm{a}}$ & $1.2^{\mathrm{b}}$ \\
\hline $72-85$ & 11 & 86 & $0.6^{\mathrm{a}}$ & $0.73^{b}$ & $0.06^{\mathrm{a}}$ & 14.1 & 59 & $7.9^{b}$ & $1.1^{\mathrm{b}}$ \\
\hline \multicolumn{10}{|l|}{ Ylistaro } \\
\hline $0-30$ & 24 & 64 & $4.6^{d}$ & $0.17^{\mathrm{a}}$ & $0.40^{d}$ & 20.5 & 9 & $10.5^{b}$ & $3.4^{\mathrm{b}}$ \\
\hline $30-52$ & 28 & 64 & $1.1^{\mathrm{ab}}$ & $0.19^{\mathrm{a}}$ & $0.19^{\mathrm{ab}}$ & 12.3 & 13 & $7.9^{\mathrm{ab}}$ & $1.1^{\mathrm{a}}$ \\
\hline $52-70$ & 27 & 67 & $1.0^{\mathrm{a}}$ & $0.17^{a}$ & $0.18^{a}$ & 9.5 & 13 & $6.1^{a}$ & $0.9^{\mathrm{a}}$ \\
\hline $70-100$ & 26 & 67 & $1.0^{\mathrm{a}}$ & $0.21^{\mathrm{a}}$ & $0.18^{\mathrm{a}}$ & 11.1 & 17 & $5.7^{a}$ & $0.9^{\mathrm{a}}$ \\
\hline $100-125$ & 28 & 65 & $1.2^{\mathrm{bc}}$ & $0.22^{a}$ & $0.22^{\mathrm{bc}}$ & 12.1 & 16 & $8.5^{\mathrm{ab}}$ & $1.0^{\mathrm{a}}$ \\
\hline $125-150$ & 26 & 65 & $1.3^{\mathrm{c}}$ & $0.31^{b}$ & $0.24^{c}$ & 13.2 & 29 & $7.8^{\mathrm{ab}}$ & $1.0^{\mathrm{a}}$ \\
\hline \multicolumn{10}{|l|}{ Laitila 1} \\
\hline $0-25$ & 43 & 53 & $6.6^{\mathrm{c}}$ & $0.22^{a}$ & $0.72^{\mathrm{c}}$ & 27.3 & 75 & $7.3^{\mathrm{c}}$ & $3.0^{f}$ \\
\hline $25-35$ & 51 & 46 & $6.6^{c}$ & $0.53^{b}$ & $0.87^{\mathrm{d}}$ & 30.4 & 13 & $17.8^{f}$ & $1.5^{b}$ \\
\hline $35-50$ & 48 & 46 & $7.6^{d}$ & $0.77^{\mathrm{c}}$ & $1.08^{e}$ & 34.2 & 15 & $16.5^{e}$ & $1.6^{\mathrm{c}}$ \\
\hline $50-90$ & 49 & 49 & $3.0^{\mathrm{a}}$ & $1.72^{e}$ & $0.44^{\mathrm{a}}$ & 49.7 & 25 & $8.6^{d}$ & $3.5^{\mathrm{e}}$ \\
\hline $90-125$ & 50 & 48 & $3.0^{\mathrm{a}}$ & $1.54^{\mathrm{b}}$ & $0.44^{\mathrm{a}}$ & 51.2 & 55 & $7.1^{\mathrm{c}}$ & $2.4^{\mathrm{d}}$ \\
\hline $125-150$ & 49 & 49 & $3.7^{b}$ & $1.68^{e}$ & $0.54^{b}$ & 51.2 & 61 & $5.9^{b}$ & $1.4^{\mathrm{b}}$ \\
\hline $150-180$ & 53 & 46 & $3.6^{\mathrm{b}}$ & $1.45^{\mathrm{d}}$ & $0.58^{\mathrm{b}}$ & 34.5 & 84 & $2.6^{\mathrm{a}}$ & $1.2^{\mathrm{a}}$ \\
\hline \multicolumn{10}{|l|}{ Laitila 2} \\
\hline $0-25$ & 48 & 50 & $4.3^{b}$ & $0.24^{\mathrm{a}}$ & $0.39^{c}$ & 23.6 & 53 & $8.7^{b}$ & $1.6^{c}$ \\
\hline $25-35$ & 53 & 44 & $2.6^{\mathrm{a}}$ & $0.38^{b}$ & $0.33^{\mathrm{a}}$ & 19.4 & 18 & $10.7^{c}$ & $1.2^{\mathrm{a}}$ \\
\hline $35-55$ & 48 & 49 & $2.7^{\mathrm{a}}$ & $0.43^{b}$ & $0.35^{b}$ & 20.2 & 16 & $12.0^{\mathrm{de}}$ & $1.2^{\mathrm{a}}$ \\
\hline $55-85$ & 48 & 50 & $2.5^{\mathrm{a}}$ & $0.44^{b}$ & $0.32^{\mathrm{a}}$ & 20.1 & 18 & $13.5^{e}$ & $1.3^{\mathrm{b}}$ \\
\hline $85-100$ & 52 & 46 & $2.7^{\mathrm{a}}$ & $0.82^{c}$ & $0.35^{\mathrm{b}}$ & 23.6 & 25 & $4.0^{\mathrm{a}}$ & $2.7^{\mathrm{d}}$ \\
\hline
\end{tabular}

${ }^{1)}$ Results of each pedon and soil characteristics were tested separately. Means marked with the same superscript do not differ at $\mathrm{P}=0.05$. Results without superscripts were not tested, owing to too few replicates.

${ }^{2)} \mathrm{NH}_{4}$-oxalate extraction, $\mathrm{pH} 3.0$

gimes. Gleyic properties, as indicated by iron (hydr)oxide cutans on ped faces, were exhibited within $50 \mathrm{~cm}$ of soil surface, meeting the requirements of Gleysols of the FAO/Unesco legend. The high contents of oxalate extractable $\mathrm{Fe}$ ( Table 3 ) are in accordance with the thick Fe hydroxide coatings on ped faces occurring in most investigated pedons.
According to Soil Taxonomy, the pedons belong to the aquic suborder of Inceptisols, Aquepts. Throughout Finland, the mean annual soil temperature at the depth of $50 \mathrm{~cm}$ is between 0 and $8^{\circ} \mathrm{C}$, and the mean summer temperature is below $15^{\circ} \mathrm{C}$ (Table 6). Thus the entire country has a cryic temperature regime (Soil Survey Staff 1996). 


\section{AGRICULTURAL AND FOOD SCIENCE IN FINLAND}

Vol. 6 (1997): 247-258.

Table 4. Morphological characteristics of the pedons.

\begin{tabular}{|c|c|c|c|c|c|c|}
\hline & $\begin{array}{l}\text { Hor- } \\
\text { izon }\end{array}$ & $\begin{array}{l}\text { Depth } \\
\mathrm{cm}\end{array}$ & $\begin{array}{l}\text { Matrix } \\
\text { colour }\end{array}$ & $\begin{array}{l}\text { Mottles and cutans }{ }^{1)} \\
\text { colour }\end{array}$ & $\begin{array}{l}\text { Text- } \\
\text { ure }^{2)}\end{array}$ & $\begin{array}{l}\text { Struct- } \\
\text { ure }^{3)}\end{array}$ \\
\hline \multirow[t]{6}{*}{ Liminka 1} & Ap & $0-25$ & $5 Y 4 / 2$ & c 7.5 YR $3 / 4$ & si & $\mathrm{gr}$ \\
\hline & Bgjl & $25-35$ & $5 Y 5 / 2$ & c 7.5 YR $3 / 4^{4)}$, f $2.5 Y 7 / 6^{4)}$ & si & If sbk \\
\hline & Bgj2 & $35-45$ & $5 Y 5 / 2$ & $\begin{array}{l}\mathrm{m} 2.5 \mathrm{Y} 7 / 6^{4)}, \mathrm{m} 7.5 \mathrm{YR} 3 / 4^{4)} \\
\text { c } 7.5 \mathrm{YR} 4 / 6^{5)}\end{array}$ & si & $2 \mathrm{f}$ sbk \\
\hline & $\mathrm{Bg}$ & $45-65$ & $5 Y R 5 / 2$ & c 7.5 YR $3 / 4^{5)}$, f $2.5 Y 7 / 6$ & si & $1 \mathrm{~m}$ sbk \\
\hline & CB & $65-85$ & 10YR 4/1 & f 10 YR $\left.3 / 6^{5}\right)$ & si & $2 \mathrm{~m}$ sbk \\
\hline & $\mathrm{Cg}$ & $85-100$ & $2.5 Y 2.5 / 1$ & f 10 YR $3 / 6^{5)}$ & si & \\
\hline \multirow[t]{4}{*}{ Liminka 2} & Ap & $0-25$ & $\begin{array}{l}\text { 10YR } 2 / 2^{6)} \\
5 Y 5 / 2^{7}\end{array}$ & c 7.5 YR $3 / 4$ & si & gr \\
\hline & Bgjl & $25-65$ & $5 Y 5 / 2$ & $\begin{array}{l}\text { c } 7.5 \text { YR } 3 / 4^{4)} \text {, c } 2.5 Y 7 / 6^{4)} \text {, } \\
\text { c } 10 \text { YR } 3 / 6^{5)}\end{array}$ & si & $1 \mathrm{~m} \mathrm{sbk}$ \\
\hline & Bgj2 & $65-72$ & 10YR 4/1 & c 2.5 Y $7 / 6^{5)}$, c 7.5 YR $3 / 4^{5)}$ & si & $1 \mathrm{~m} \mathrm{sbk}$ \\
\hline & $\mathrm{CBg}$ & $72-85$ & $2.5 Y 2.5 / 1$ & & si & $2 \mathrm{c} \mathrm{sbk}$ \\
\hline \multirow[t]{5}{*}{ Ylistaro } & Ap & $0-30$ & \multicolumn{2}{|c|}{$7.5 \mathrm{YR} 2 / 2,10 \mathrm{YR} 6 / 2^{8)}$} & sil & $1 \mathrm{~m}$ sbk \\
\hline & $\mathrm{Bw}$ & $30-52$ & $5 Y 5 / 1$ & c $7.5 \mathrm{YR} 4 / 4^{4), 5)}$ & sil & $2 \mathrm{~m} \mathrm{sbk}$ \\
\hline & Bgjl & $52-70$ & $5 Y 5 / 1$ & m 7.5YR 3/4 4 , 5), c 2.5 Y 8/4 4 , 5) & sil & $2 \mathrm{~m}$ sbk \\
\hline & Bgj2 & $70-100$ & $5 Y 5 / 1$ & m 5 YR 3/3 $3^{4) .5)}, 2.5 Y 8 / 4^{4 / 5)}$ & sil & $2 \mathrm{c} \mathrm{pr}$ \\
\hline & Bgj3 & $100-150$ & 5 Y $5 / 1$ & m 7.5YR 3/4 ${ }^{4), 5)}, \mathrm{m} 2.5 \mathrm{Y} 8 / 4^{4), 5)}$ & sil & $2 \mathrm{vc} \mathrm{pr}$ \\
\hline \multirow[t]{6}{*}{ Laitila 1} & Ap & $0-25$ & \multicolumn{2}{|c|}{$2.5 Y 3 / 2,2.5 Y^{6} 6 / 2^{8)}$} & sic & $1 \mathrm{~m}$ sbk \\
\hline & $\mathrm{AB}$ & $25-35$ & $2.5 \mathrm{Y} 4 / 2$ & m 10YR 3/4 & sic & $2 \mathrm{~m} \mathrm{pl}$ \\
\hline & Bgl & $35-50$ & $2.5 \mathrm{Y} 4 / 2$ & m 7.5YR 4/(65), m 5YR 3/4 & sic & $3 \mathrm{~m} \mathrm{pr}$ \\
\hline & Bg2 & $50-90$ & $2.5 \mathrm{Y} 4 / 2$ & m 7.5YR 4/65), m 5YR 2.5/25) & sic & $3 \mathrm{c} \mathrm{pr}$ \\
\hline & $\mathrm{BCg}$ & $90-125$ & $2.5 Y 4 / 1$ & c 7.5 YR $4 / 6^{5)}$ & sic & $2 \mathrm{c} \mathrm{pr}$ \\
\hline & $\mathrm{C}$ & $125-180$ & $2.5 Y 4 / 1$ & & sic & massive \\
\hline \multirow[t]{5}{*}{ Laitila 2} & Ap & $0-25$ & \multicolumn{2}{|c|}{$2.5 Y 3 / 2,2.5 Y^{2} 6 / 2^{8)}$} & sic & $1 \mathrm{~m}$ sbk \\
\hline & $\mathrm{AB}$ & $25-35$ & $2.5 \mathrm{Y} 4 / 2$ & $\mathrm{~m} \mathrm{10YR} 3 / 4^{4), 5)}$, vf $2.5 \mathrm{Y} 7 / 6$ & sic & $2 \mathrm{~m}$ sbk \\
\hline & Bgl & $35-55$ & $2.5 Y 4 / 2$ & $\begin{array}{l}\text { m } 7.5 \text { YR } 4 / 6^{5)} \text {, m 5YR 3/45), } \\
\text { vf } 2.5 \text { Y } 7 / 6\end{array}$ & sic & $3 \mathrm{~m} \mathrm{pr}$ \\
\hline & Bg2 & $55-85$ & $2.5 \mathrm{Y} 4 / 2$ & m 7.5YR 4/65), m 5YR 2.5/25) & sic & $3 \mathrm{c} \mathrm{pr}$ \\
\hline & $\mathrm{BCg}$ & $85-100$ & $2.5 \mathrm{Y} 4 / 1$ & c 7.5 YR $4 / 6^{5)}$, f 2.5 Y $2.5 / 1^{9)}$ & sic & $3 \mathrm{c} \mathrm{pr}$ \\
\hline
\end{tabular}

1) $\mathrm{m}=$ many, $>20 \%$; $\mathrm{c}=$ common, $2-20 \%$; $\mathrm{f}=\mathrm{few},<2 \%$

2) $\mathrm{si}=$ silt, sil=silt loam, sic=silty clay

3) 1 =weak, $2=$ moderate, $3=$ strong, $\mathrm{f}=$ fine, $\mathrm{m}=$ medium, $\mathrm{c}=$ coarse, $\mathrm{v}=$ =very coarse gr=granular, sbk=subangular blocky, $\mathrm{pl}=$ platy, $\mathrm{pr}=$ prismatic

4) around previous root channels, yellow zone between dark brown zone

5) on ped faces

${ }^{6)}$ organic matter and ${ }^{7}$ mineral material not completely mixed

8) dry color

9) interiors in peds

Both Liminka pedons had horizons that had a $\mathrm{pH} \leq 3.5$ (Fig. 4), a $\mathrm{SO}_{4}-\mathrm{S}$ content above $0.05 \%$ (Table 5) and yellow jarosite mottles at the 30 $65 \mathrm{~cm}$ depth (Table 4), which are the criteria of sulfuric horizons. Because they occurred within
$50 \mathrm{~cm}$ of soil surface, these pedons classify as coarse silty, cryic Typic Sulfaquepts (Soil Survey Staff 1996) and medium-textured Thionic Gleysols (FAO 1988). Both Liminka pedons also have sulfidic materials below the acidic horizons 
Yli-Halla, M. Acid sulphate soils of Finland

Table 5. Soil $\mathrm{pH}$ and $\mathrm{SO}_{4}-\mathrm{S}$ content of the pedons. ${ }^{1)}$

\begin{tabular}{llll}
\hline $\begin{array}{l}\text { Depth } \\
\mathrm{cm}\end{array}$ & $\begin{array}{l}\mathrm{pH} \\
\text { moist }\end{array}$ & $\begin{array}{l}\mathrm{pH} \\
\text { dried }\end{array}$ & $\begin{array}{l}\mathrm{SO}_{4}-\mathrm{S}^{2} \\
\mathrm{mg} / \mathrm{kg}\end{array}$ \\
\hline $\begin{array}{l}\text { Liminka 1 } \\
0-25\end{array}$ & $6.5^{\mathrm{c}}$ & $6.1^{\mathrm{a}}$ & $623^{\mathrm{c}}$ \\
$25-35$ & $4.7^{\mathrm{abc}}$ & $4.0^{\mathrm{b}}$ & $271^{\mathrm{a}}$ \\
$35-45$ & $3.9^{\mathrm{ab}}$ & $3.6^{\mathrm{d}}$ & $323^{\mathrm{b}}$ \\
$45-65$ & $3.4^{\mathrm{a}}$ & $3.4^{\mathrm{e}}$ & $655^{\mathrm{d}}$ \\
$65-85$ & $5.3^{\mathrm{bc}}$ & $3.5^{\mathrm{e}}$ & $1325^{\mathrm{f}}$ \\
$85-100$ & $6.5^{\mathrm{c}}$ & $3.9^{\mathrm{c}}$ & $1106^{\mathrm{c}}$ \\
Liminka 2 & & & \\
$0-25$ & $5.6^{\mathrm{b}}$ & $5.7^{\mathrm{a}}$ & $854^{\mathrm{b}}$ \\
$25-65$ & $3.4^{\mathrm{a}}$ & $3.4^{\mathrm{b}}$ & $661^{\mathrm{a}}$ \\
$65-72$ & $4.6^{\mathrm{ab}}$ & $2.9^{\mathrm{c}}$ & $2442^{\mathrm{d}}$ \\
$72-85$ & $5.4^{\mathrm{b}}$ & $3.4^{\mathrm{b}}$ & $1496^{\mathrm{c}}$ \\
90 & $6.0^{\mathrm{b}}$ & n.d. & n.d.
\end{tabular}

Ylistaro

$\begin{array}{lllr}0-30 & 4.3^{\mathrm{e}} & 4.2^{\mathrm{d}} & 29^{\mathrm{a}} \\ 30-52 & 3.9^{\mathrm{d}} & 3.7^{\mathrm{c}} & 42^{\mathrm{b}} \\ 52-70 & 3.8^{\mathrm{c}} & 3.5^{\mathrm{c}} & 60^{\mathrm{c}} \\ 70-100 & 3.5^{\mathrm{b}} & 3.3^{\mathrm{ab}} & 112^{\mathrm{d}} \\ 100-125 & 3.4^{\mathrm{a}} & 3.3^{\mathrm{a}} & 156^{\mathrm{c}} \\ 125-150 & 3.4^{\mathrm{a}} & 3.4^{\mathrm{abc}} & 480^{\mathrm{f}}\end{array}$

Laitila 1

$\begin{array}{lllr}0-25 & 5.9^{c} & 5.7^{\mathrm{e}} & 222^{\mathrm{a}} \\ 25-35 & 3.5^{\mathrm{ab}} & 3.3^{\mathrm{c}} & 413^{\mathrm{b}} \\ 35-50 & 3.3^{\mathrm{a}} & 3.0^{\mathrm{b}} & 1410^{\mathrm{c}} \\ 50-90 & 3.8^{\mathrm{b}} & 2.8^{\mathrm{a}} & 5777^{\mathrm{f}} \\ 90-125 & 6.0^{\mathrm{c}} & 3.1^{\mathrm{bc}} & 4329^{\mathrm{e}} \\ 125-150 & 7.6^{\mathrm{d}} & 3.0^{\mathrm{ab}} & 4250^{\mathrm{c}} \\ 150-180 & 7.5^{\mathrm{d}} & 3.7^{\mathrm{d}} & 3394^{\mathrm{d}}\end{array}$

Laitila 2

\begin{tabular}{lllr}
$0-25$ & $5.3^{\mathrm{c}}$ & $5.1^{\mathrm{a}}$ & $61^{\mathrm{a}}$ \\
$25-35$ & $3.7^{\mathrm{b}}$ & $3.8^{\mathrm{b}}$ & $156^{\mathrm{b}}$ \\
$35-55$ & $3.4^{\mathrm{ab}}$ & $3.5^{\mathrm{c}}$ & $227^{\mathrm{c}}$ \\
$55-85$ & $3.2^{\mathrm{a}}$ & $3.4^{\mathrm{c}}$ & $462^{\mathrm{d}}$ \\
$85-100$ & $3.6^{\mathrm{b}}$ & $3.3^{\mathrm{c}}$ & $2069^{\mathrm{c}}$ \\
\hline
\end{tabular}

1) Results of each soil and soil characteristics were tested separately. Means marked with the same superscript do not differ at $\mathrm{P}=0.05$.

2) Incubated samples, $0.01 \mathrm{M} \mathrm{CaCl}_{2}$ extraction

n.d. $=$ not determined

(below $65 \mathrm{~cm}$ ), as indicated by the drop in $\mathrm{pH}$ upon drying (Table 5). These black layers had negative redox potentials (Fig. 2) and the colour of iron monosulfide although these layers did not
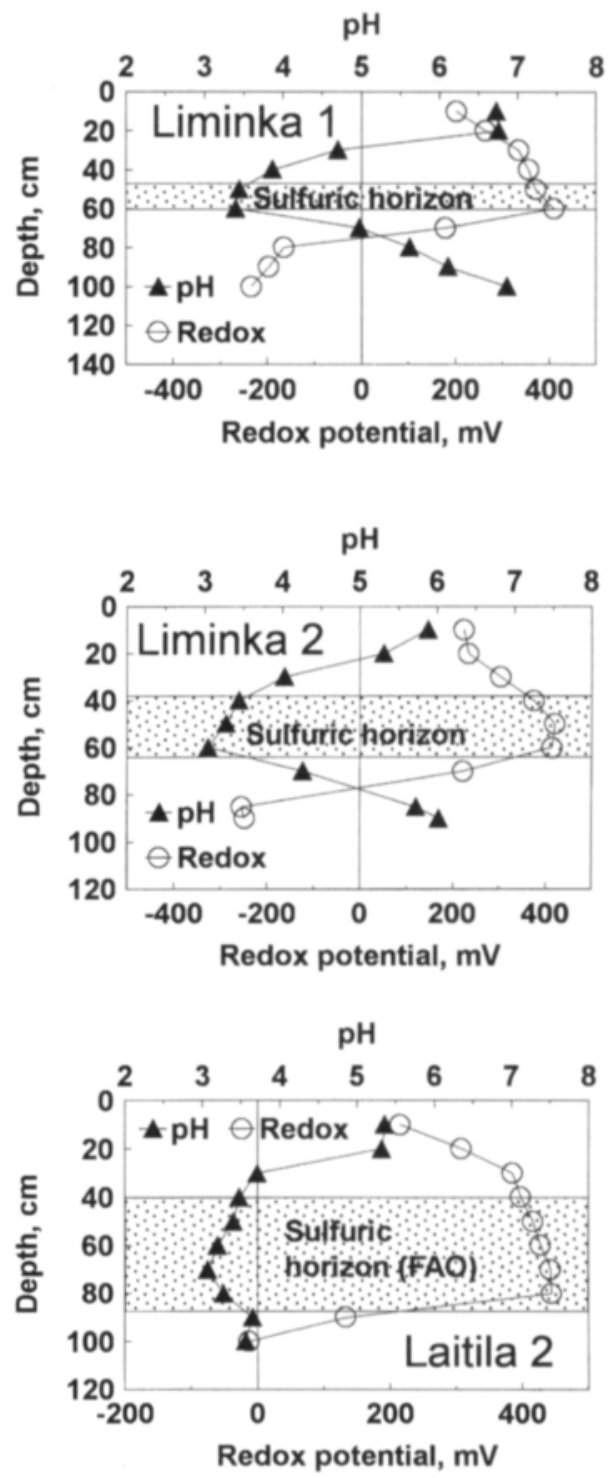

Fig. 4. Soil pH and redox potential of the Liminka 1 and 2 and Laitila 2 pedons, measured in the soil pit.

meet the total S requirement $(0.75 \%)$ of sulfidic materials in the FAO/Unesco legend.

The Ylistaro pedon was oxidized throughout the sampling depth as indicated by the low $\mathrm{pH}$ 
Vol. 6 (1997): 247-258.

Table 6. Soil temperatures in Finland, measured at the depth of $50 \mathrm{~cm}$.

\begin{tabular}{lllll}
\hline Location & & Period & $\begin{array}{l}\text { Mean } \\
\text { annual } \\
{ }^{\circ} \mathrm{C}\end{array}$ & $\begin{array}{l}\text { Mean } \\
\text { summer } \\
{ }^{\circ} \mathrm{C}\end{array}$ \\
\hline Jokioinen & $60^{\circ} 49^{\prime} \mathrm{N}, 23^{\circ} 30^{\prime} \mathrm{E}$ & $1957-1970$ & $5.9^{1)}$ & $12.7^{1)}$ \\
Ylistaro & $62^{\circ} 57^{\prime} \mathrm{N}, 22^{\circ} 31^{\prime} \mathrm{E}$ & $1968-1980$ & $5.5^{2)}$ & $13.1^{2)}$ \\
Sodankylä & $67^{\circ} 22^{\prime} \mathrm{N}, 26^{\circ} 39^{\prime} \mathrm{E}$ & $1963-1970$ & $3.0^{1)}$ & $11.8^{1)}$ \\
Utsjoki & $69^{\circ} 45^{\prime} \mathrm{N}, 27^{\circ} 02^{\prime} \mathrm{E}$ & $1964-1970$ & $1.9^{1)}$ & $6.2^{1)}$ \\
\hline
\end{tabular}

1) Finnish Meteorological Institute (1979)

2) Agricultural Research Centre of Finland, unpublished data

which did not change upon aerobic incubation (Table 5). Long-term drainage has lead to leaching of $\mathrm{SO}_{4}-\mathrm{S}$ to below the requirement for the sulfuric horizon in Soil Taxonomy. However, below $52 \mathrm{~cm}$, jarosite was present (Table 4), an alternative criterion of the sulfuric horizon, and below $100 \mathrm{~cm}$ the $\mathrm{pH}$ was $<3.5$ (Table 5 ). Thus a sulfuric horizon was present at $100-150 \mathrm{~cm}$. The Ylistaro pedon is classified as a fine silty Sulfic Cryaquept (Soil Survey Staff 1996). In the FAO/ Unesco legend, the $\mathrm{pH}<3.5$ is sufficient to identify a sulfuric horizon, and this pedon classifies as a medium-textured Thionic Gleysol (FAO 1988).

The Laitila 1 pedon had a $\mathrm{pH}<3.5$ and a high content of $\mathrm{SO}_{4}-\mathrm{S}$ in the $35-50 \mathrm{~cm}$ layer, which qualifies as a sulfuric horizon (Table 5). Very little or no jarosite was observed. At $50-150 \mathrm{~cm}$ there were sulfidic materials according to the requirements of both classification systems (Table 2). The pedon classifies as a fine, cryic Typic Sulfaquept (Soil Survey Staff 1996) and a fine textured Thionic Gleysol (FAO 1988).

In the Laitila 2 pedon there was a layer at $40-85 \mathrm{~cm}$ which had a $\mathrm{pH}<3.5$ in moist soil (Fig. 4). None of the other requirements of a sulfuric horizon of Soil Taxonomy were fulfilled: neither jarosite nor sulfidic materials occurred within the sampling depth, and the content of $\mathrm{SO}_{4}-\mathrm{S}$ was too low. However, at $85-100 \mathrm{~cm}$ there was a $\mathrm{SO}_{4}$-S content $>0.05 \%$ and a $\mathrm{pH}<4.0$ (Table 5), allowing the soil to be classified as a fine Sulfic
Cryaquept (Soil Survey Staff 1996). Moreover, the redox potential reached a value of $0 \mathrm{mV}$ at approximately $100 \mathrm{~cm}$ (Fig. 2). Sulfidic materials can be expected below this depth, supporting the above classification. According to the FAO/Unesco legend, a sulfuric horizon occurs at $40-85 \mathrm{~cm}$ in this soil. Therefore, Laitila 2 is a fine-textured Thionic Gleysol.

\section{Discussion}

Major processes after drainage of soils formed in sulfidic sediments include 1) oxidation of sulfidic materials to sulfuric acid with a consequent decrease of $\mathrm{pH}$ and 2) leaching of watersoluble components (sulphate accompanied by acid and base cations), resulting in the decrease of base saturation. The pedons of this study can be arranged according to decreasing base saturation and decreasing contents of sulfidic materials and sulphate as follows:

Laitila $1>$ Liminka $2>$ Liminka $1>$ Laitila 2 $>$ Ylistaro

The Liminka 1 and 2 and Laitila 1 pedons (Typic Sulfaquepts/Thionic Gleysols) contained sulfidic materials and sulfuric horizons with high contents of easily soluble $\mathrm{SO}_{4}-\mathrm{S}$. The Laitila 1 


\section{AGRICULTURAL AND FOOD SCIENCE IN FINLAND}

\section{Yli-Halla, M. Acid sulphate soils of Finland}

pedon is placed first because it is the only pedon which contained sulfidic materials also according to the criteria of the FAO/Unesco legend. The sulfuric horizon was closer to the soil surface in the Liminka 2 pedon than in the Liminka 1 pedon and therefore it has more pronounced a.s. characteristics than the Liminka 1 pedon. The Ylistaro and Laitila 2 pedons (Sulfic Cryaquepts/ Thionic Gleysols) were oxidized and leached to greater depths, resulting in lower base saturation. The Laitila 2 pedon had a sulfuric horizon only according to the criteria of the FAO/Unesco legend. It was much more oxidized and leached than the Laitila 1 pedon nearby, because the Laitila 2 pedon was slightly higher in the landscape while the Laitila 1 pedon was taken from the more poorly drained deepest point of the polder. However, owing to the lower base saturation and $\mathrm{SO}_{4}-\mathrm{S}$ content, the Ylistaro pedon can be considered the most leached one and is placed last in the sequence.

According to this investigation, cultivated a.s. soils along the western coast of Finland commonly qualify as Typic Sulfaquepts (Soil Survey Staff 1996). The soils of Mälsor (Koivulahti), Bäckby (Ähtävä) and Vaasa in Southern and Central Ostrobothnia (Hartikainen and Yli-Halla 1986, Palko et al. 1987, and Erviö 1991, respectively) probably also meet the requirements of Typic Sulfaquepts. The present study and previous investigations suggest that the soils of Storsjö (Lapväärtti) in Southern Ostrobothnia (Hartikainen and Yli-Halla 1986, Palko and Saari 1987) and many soils in the Sirppujoki catchment in Southwestern Finland (Palko et al. 1985) can be classified as Sulfic Cryaquepts. The occurrence of Sulfic Cryaquepts in Finland, in addition to the Swedish soils (Öborn 1989), justifies the recognition of this taxon which was added only in 1992.

Pedogenesis results in gradual changes in the soils formed in sulfidic sediments. Besides artificial drainage, pedogenic transformations in a.s. soils are promoted also by the postglacial land uplift which continues at the annual rate of 4-8 $\mathrm{mm}$ on the western coast of Finland. The present pedons constitute a sequence exemplifying dif- ferent stages of pedogenesis of a.s. soils. Initially, all of them were probably Sulfaquents, developing into Sulfaquepts when drained for agriculture. Over time the sulfuric horizon moves downwards and a Sulfaquept is transformed into a Sulfic Cryaquept like in the Ylistaro pedon which exhibits a sulfuric horizon below $100 \mathrm{~cm}$. That soil probably once had a sulfuric horizon below $50 \mathrm{~cm}$ because there was still abundant jarosite at that depth. The Ylistaro pedon resembles the two Sulfic Cryaquepts (Ängesby and Ersnäs) from Northern Sweden (Öborn 1989) which contained jarosite but were low in $\mathrm{SO}_{4}-\mathrm{S}$ and had the most acidic horizons at the depth of $70-110 \mathrm{~cm}$.

Principally, a similar sequence as described above was presented by Fanning and Fanning (1989) in an East-Texas environment where a Sulfaquent developed into a Sulfaquept and further into a Sulfic Endoaquept and finally into a Haplustalf and a Vertic Albaqualf. In the cool and humid climate of Finland, cultivated a.s. soils are eventually transformed from Sulfic Cryaquepts to Typic Cryaquepts (Soil Survey Staff 1996). In terms of the FAO/Unesco system, Thionic Gleysols become Dystric Gleysols or Gleyic Cambisols. The Härkmeri soil (Lapväärtti) in Southern Ostrobothnia (Yli-Halla and Hartikainen 1984) represents this final stage. It had a $\mathrm{pH}\left(\mathrm{CaCl}_{2}\right)$ of 3.5 in aerobic subsoil, but none of the other requirements of the a.s. soils were met. Typically, low $\mathrm{pH}$ is a more persistent soil characteristic than a high $\mathrm{SO}_{4}-\mathrm{S}$ content.

In a recent survey (Puustinen et al. 1994), approximately 300,000 ha of cultivated soils in Finland with a minimum $\mathrm{pH}<5$ in subsoil were recognized as a.s. soils. This estimate is certainly much greater than the area of soils meeting the requirements of a.s. soils as stated in Soil Taxonomy or the FAO/Unesco Soil Map of the World. The national criteria can also be questioned from the environmental point of view. Based on the above results, it can be concluded that soils with subsoil $\mathrm{pH}$ values of 4-5 (a.s. soils according to the national criteria) do not contain actively oxidising sulfide or appreciable 


\section{AGRICULTURAL AND FOOD SCIENCE IN FINLAND}

Vol. 6 (1997): 247-258.

quantities of acidic solutes. These soils may have passed the raw, acid-generating phase or their sulfide content was too low in the first place to produce sufficient sulfuric acid to lower the $\mathrm{pH}$ below 4.0. Therefore, soils with subsoil $\mathrm{pH}$ values between 4 and 5 do not present a hazard of acid loading to the recipient water courses. If these soils are included in a.s. soils, the environmental problems caused by a.s. soils in Finland are apparently overemphasized and may draw attention of authorities away from the re- mediation of the acid loading in areas with the highest loading potential.

Acknowledgements. The author thanks Dr. Jukka Palko from the North Ostrobothnia Regional Environment Centre in Oulu for helping locate and sample the representative pedons in Liminka and Laitila, Mr. Antti Palttila and Mrs. Mirja Palttila, M.Sc., for helping sample the Laitila pedons and Dr. Delvin S. Fanning from the University of Maryland, USA, Dr. Delbert L. Mokma from Michigan State University, USA, and Dr. David Dent from the University of East Anglia, England, for reviewing this manuscript.

\section{References}

Dent, D. 1986. Acid sulphate soils: a baseline for research and development. ILRI Publication 39. Wageningen, The Netherlands. 204 p.

Erviö, R. 1975. Kyrönjoen vesistöalueen rikkipitoiset viljelysmaat. Summary: Cultivated sulphate soils in the drainage basin of river Kyrönjoki. Journal of the Scientific Agricultural Society of Finland 47: 550- 561.

- 1991. Chemical properties of air-dried samples from an unlimed and limed acid sulphate soil profile and leaching of elements from the profiles. Annales Agriculturae Fenniae 30: 221-229.

- \& Palko, J. 1984. Macronutrient and micronutrient status of cultivated acid sulphate soils at Liminka, Finland. Annales Agriculturae Fenniae 23: 121-134.

Fanning, D.S. \& Fanning, M.C.B. 1989. Soil: morphology, genesis and classification. John Wiley and Sons. 395 p. New York.

- \& Witty, J.E. 1993. Revisions of Soil Taxonomy for acid sulphate soils. In: Dent, D.L. \& Mensvoort, M.E.F. van (eds.). Selected papers of the Ho Chi Minh City symposium on acid sulphate soils. ILRI Publication 53: 61-69.

FAO 1988. FAO/Unesco Soil Map of the World. Revised legend, with corrections. World Resources Report 60, FAO, Rome. Reprinted as Technical Paper 20, ISRIC, Wageningen, The Netherlands, 1994. 140 p.

Finnish Meteorological Institute. 1979. Results of soil temperature measurements in Finland 1961-1970. Soil temperature measurements $3.59 \mathrm{p}$.

Hartikainen, H. \& Yli-Halla, M. 1986. Oxidation-induced leaching of sulphate and cations from acid sulphate soils. Water, Air and Soil Pollution 27: 1-13.

Kivinen, E. 1938. Über die Eigenschaften der Gyttjaböden. Bodenkunde und Pflanzenernăhrung 9-10: $122-134$.

McKeague, J.A. \& Day, J.H. 1966. Dithionite- and oxalateextractable $\mathrm{Fe}$ and $\mathrm{Al}$ as aids in differentiating various classes of soils. Canadian Journal of Soil Science 46: 13-22.
Mensvoort, M.E.F. van \& Dent, D. 1997. Acid sulphate soils. Advances in Soil Science 22: 301-335.

Öborn, I. 1989. Properties and classification of some acid sulphate soils in Sweden. Geoderma 45: 197-219.

- 1994. Morphology, chemistry, mineralogy and fertility of some acid sulfate soils in Sweden. Swedish University of Agricultural Sciences. Department of Soil Science. Reports and dissertations 18. 65 p. Ph.D. Thesis.

Palko, J. 1988. Happamien sulfaattimaiden kuivatus ja kalkitus Limingan koekentāllă 1984-87. Summary: Drainage and liming of acid sulphate soils in the polder in Liminka, Finland 1984-87. National Board of Waters and Environment. Publications 19. 86 p.

- 1994. Acid sulphate soils and their agricultural and environmental problems in Finland. Acta Universitatis Ouluensis C. Technica 75. 58 p. Ph.D Thesis.

- , Răsănen, M. \& Alasaarela, E. 1985. Happamien sulfaattimaiden esiintyminen ja vaikutus veden laatuun Sirppujoen vesistōalueella. National Board of Waters. Report 260. 95 p.

- , Răsănen, M. \& Alasaarela, E. 1987. Luodon-Öjanjärven valuma-alueen maaperän ja vesistön happamuuskartoitus. Summary: The survey of the soil and water acidity in the catchment area of Luodon-Ojanjărvi. National Board of Waters and Environment. Publications 11: 61-100.

- \& Saari, M. 1987. Lapväärtin-Isojoen vesistőalueella sijaitsevan Storsjön jărvikuivion happamat sulfaattimaat. Summary: Acid sulphate soils in the drained lake area or Storsjö in the drainage basin of river Lapväărtti-Isojoki. National Board of Waters and Environment. Publications 11: 3-22.

- \& Yli-Halla, M. 1993. Assessment and managament of acidity release upon drainage of acid sulphate soils in Finland. In: Dent, D.L. \& Mensvoort, M.E.F. van (eds.). Selected papers of the Ho Chi Minh City symposium on acid sulphate soils. ILRI Publication 53: 411-418. 


\title{
AGRICULTURAL AND FOOD SCIENCE IN FINLAND
}

Yli-Halla, M. Acid sulphate soils of Finland

Pons, L.J. 1973. Outline of the genesis, characteristics, classification and improvement of acid sulfate soils. In: Dost, H. (ed.). Proceedings of the international symposium on acid sulphate soils. ILRI Publications 18, I: 3-27.

Purokoski, P. 1958. Die schwefelhaltigen Tonsedimente in dem flachlandgebiet von Liminka im Lichte chemischer Forschung. Agrogeologische Publicationen 70. 85 p. Helsinki.

- 1959. Rannikkoseudun rikkipitoisista maista. Referat: Über die schwefelhaltigen Böden an der Küste Finnlands. Agrogeologische Publicationen 74. 27 p. Helsinki.

Puustinen, M., Merilä, E., Palko, J. \& Seuna, P. 1994.
Kuivatustila, viljelykäytăntő ja vesistőkuormitukseen vaikuttavat ominaisuudet Suomen pelloilla. Summary: Drainage level, cultivation practices and factors affecting load on waterways in Finnish farmland. National Board of Waters and Environment. Report 198. $323 \mathrm{p}$.

Soil Survey Staff 1996. Keys to Soil Taxonomy. 7th ed. USDA. Natural Resources Conservation Service. U.S. Government Printing Office. 644 p.

Yli-Halla, M. \& Hartikainen, H. 1984. Rikin, raudan, alumiinin ja mangaanin huuhtoutuminen kolmen happaman sulfaattimaaprofiilin kerrosnäytteistä. Vesihallituksen monistesarja 35. Helsinki. 40 p.

\section{SELOSTUS}

\section{Suomen happamien sulfaattimaiden kansainvälinen luokittelu}

\author{
Markku Yli-Halla \\ Maatalouden tutkimuskeskus
}

Suomessa luokitellaan happamiksi sulfaattimaiksi sellaiset maat, joissa kuivaamattomasta näytteestä mitattu maaprofiilin minimi-pH on alle 5 ja maan hapetus-pelkistyspotentiaali ensin nousee ja sitten laskee mentäessä maaprofiilissa alaspäin. Kansainvälisiä maaperän luokitusjärjestelmiä ovat FAOn/Unescon järjestelmä ja Yhdysvalloissa kehitetty Soil Taxonomy -järjestelmä. Jotta maa olisi sulfaattimaa, sen $\mathrm{pH}: \mathrm{n}$ on oltava Soil Taxonomy-järjestelmässä alle 4,0 ja FAOn/Unescon järjestelmässä alle 3,5. Vaihtoehtoisesti pelkistyneessä maassa on oltava niin paljon sulfidia, että hapettuvan maan $\mathrm{pH}$ laskee alle e.m. raja-arvojen. Luokittelurajojen tarkoituksena on, että (happamiksi) sulfaattimaiksi nimettäisiin vain sellai- sia maita, joissa on tai joissa voi kehittyä runsaasti happamuutta. Tällaiset maat voivat tuottaa ympäristöönsä merkittävää happamoittavaa kuormitusta.

Tässä tutkimuksessa Limingasta, Ylistarosta ja Laitilasta otettuja happamia sulfaattimaita luokiteltiin kansainvälisten kriteerien mukaan. Maaprofiilien tutkimus osoitti, että maamme länsirannikolla esiintyy yleisesti Soil Taxononomy -järjestelmän Typic Sulfaquepts- ja Sulfic Cryaquepts- luokkiin ja FAOn/Unescon luokituksen Thionic Gleysols- luokkiin kuuluvia viljelymaita. Sellaiset Suomessa happamiksi sulfaattimaiksi nimitetyt maat, joiden $\mathrm{pH}$ on 4-5, eivät kansainvälisten kriteerien mukaan kuitenkaan ole happamia sulfaattimaita. 